\title{
COMUNICAÇÃO INTESTINO E CÉREBRO: REVISÃO DE LITERATURA
}

\author{
Mileny Costa de Almeida*1; Gustavo Garcia Pacheco²; Ingrid Fortunato Garcia de Carvalho²; \\ Lorrane Machado Mendes²; Leonardo Garcia Pacheco²; Gabriel Bianchi da Silva²; \\ Raquel Santos Berto de Faria ${ }^{3}$
}

${ }^{1}$ Discente de Medicina, Centro Universitário de Goiatuba (UNICERRADO); ${ }^{2}$ Discente de Medicina, Universidade de Franca (UNIFRAN); ${ }^{3}$ Docente de Medicina, Centro Universitário de Goiatuba (UNICERRADO)

\begin{tabular}{|c|c|}
\hline ARTICLE INFO & ABSTRACT \\
\hline Article History: & \multirow{10}{*}{$\begin{array}{l}\text { Introdução: } O \text { ambiente intestinal é colonizado por trilhões de microrganismos, os quais vivem } \\
\text { de forma comensal e simbiótica. Esse microbioma exerce importantes papéis na promoção da } \\
\text { saúde, tanto no intestino, quanto em ambientes extra-intestinais, como sistema nervoso central e } \\
\text { sistema imune. Materiais e métodos: Foi realizada uma revisão qualitativa e integrativa a partir } \\
\text { de fontes encontradas na plataforma PubMed, publicadas nos últimos cinco anos e que } \\
\text { relacionavam a microbiota intestinal com o sistema imune e nervoso. Resultados e Discussão: A } \\
\text { falha na interação do eixo intestino-cérebro pode ocasionar distúrbios da inflamação, doenças } \\
\text { autoimunes, cardiovasculares, psiquiátricas, neurológicas, respostas anormais ao estresse, } \\
\text { comportamentos alterados e distúrbios metabólicos. Essa disbiose pode ter influência ambiental, } \\
\text { dietética e medicamentosa. Conclusões: O eixo intestino-cérebro influencia diretamente a } \\
\text { homeostase do organismo, seja de forma positiva - com seu bom funcionamento -, seja } \\
\text { negativamente - com sua desregulação propiciando patologias. Faz-se necessária a realização de } \\
\text { mais estudos sobre o microbioma e sua influência no bom funcionamento do organismo e na } \\
\text { prevenção de doenças. }\end{array}$} \\
\hline Received $10^{\text {th }}$ May, 2021 & \\
\hline Received in revised form & \\
\hline $20^{\text {th }}$ June, 2021 & \\
\hline $\begin{array}{l}\text { Accepted } 19^{\text {th }} \text { July, } 2021 \\
\text { Published online } 29^{\text {th }} \text { August, } 2021\end{array}$ & \\
\hline & \\
\hline Key Words: & \\
\hline $\begin{array}{l}\text { Microbiota; Sistema imune; } \\
\text { Sistema Nervoso; Patologias. }\end{array}$ & \\
\hline *Corresponding author: & \\
\hline Mileny Costa de Almeida, & \\
\hline
\end{tabular}

Copyright (C) 2021, Mileny Costa de Almeida et al., This is an open access article distributed under the Creative Commons Attribution License, which permits unrestricted use, distribution, and reproduction in any medium, provided the original work is properly cited.

Citation: Mileny Costa de Almeida; Gustavo Garcia Pacheco; Ingrid Fortunato Garcia de Carvalho; Lorrane Machado Mendes; Leonardo Garcia Pacheco; Gabriel Bianchi da Silva; Raquel Santos Berto de Faria. 2021. "Comunicação intestino e cérebro: revisão de literatura", International Journal of Development Research, 11, (08), 49689-49691.

\section{INTRODUÇÃO}

O trato gastrointestinal dos humanos é colonizado por trilhões de microrganismos - bactérias, fungos e vírus -, em que são chamados, coletivamente, de microbiota intestinal (FUNG, 2020). A comunidade microbiana inclui micróbios comensais e simbióticos, o que reflete a coevolução do hospedeiro e seu microbioma (FITZGIBBON; MILLS, 2020). A relação entre microbioma e o hospedeiro é mutuamente benéfica, enquanto o hospedeiro fornece um ambiente de sobrevivência e proliferação, os micróbios contribuem para processos importantes na fisiologia humana (ARAOS; D'AGATA, 2019; FITZGIBBON; MILLS, 2020). As funções da microbiota intestinal podem ser agrupadas, resumidamente, em proteção, imunidade e metabolismo (RAMIREZ, 2017). A microbiota intestinal protege o tecido por bloquear as bactérias patogênicas que geram um desequilíbrio no ambiente intestinal, ocasionando doenças locais e ou sistêmicas (SOARES, 2019). Além da função antibacteriana, a relação microbiota-hospedeiro tem como função imunomoduladora, metabolismo nutricional, reestruturação da mucosa intestinal, auxílio no sistema autoimune, regulação da agregação de gordura corporal, degradação de alimentos, extração de energia e produção de conteúdos importantes, como vitamina $\mathrm{K}$, cobalamina (B12), ácido fólico, biotina e ácido pantotênico (FUNG, 2020). O microbioma intestinal funciona como um órgão endócrino, pois exerce efeitos em locais remotos da origem, como pulmão e cérebro (FITZGIBBON; MILLS, 2020). O eixo intestino-cérebro, que tem como função integrar centros cerebrais com sistema nervoso entérico, é formado por complexas vias neurais - aferentes e eferentes - e gânglios, englobando os sistemas nervoso central, nervoso entérico, nervoso autônomo, imune e endócrino (ZORZO, 2017). Os produtos derivados do microbioma humano, como lipopolissacarídeos, proteínas de ligação, peptideoglicanos são importantes no eixo supracitado (RUFF; GREILING; KRIEGEL, 2020; SUN; LI; NIE, 2020). A interação bidirecional no eixo intestino-cérebro permite que o cérebro influencie o trato gastrointestinal, e a microbiota intestinal exerça influência sobre o sistema nervoso central (SNC) (SUN; LI; NIE, 2020). Os micróbios intestinais têm efeitos de longo alcance em função das células imunes residentes no sistema nervoso central (FUNG, 2020). O ecossistema intestinal integra o mecanismo de homeostase corporal, de forma que distúrbios da microbiota implicam na gênese de saúde e doença (ZORZO, 2017). A falha nessa interação pode ocasionar distúrbios da inflamação, respostas anormais ao 
estresse, comportamentos alterados e distúrbios metabólicos (SUN; LI; NIE, 2020). O objetivo do presente trabalho é compreender a relação do microbioma intestinal com o funcionamento intestinocérebro e a homeostase.

\section{MATERIAIS E MÉTODOS}

Foi realizada uma pesquisa integrativa, quantitativa e exploratória de revisão bibliográfica na plataforma PubMed (Serviço da National Library of Medicine), durante o mês de maio de 2021, com os seguintes descritores: [gut microbiota] e [microbiota-gut-brain]. O estudo utiliza referências bibliográficas e científicas a fim de entender o eixo intestino-cérebro e as colaborações extra-intestinais da microbiota. Foram filtrados os trabalhos científicos dos últimos cinco anos, dos 158 resultados, foram selecionados os artigos científicos que mais se encaixavam na vertente do tema. $O$ processo de seleção do estudo constituiu: 1) análise de títulos;2) análise dos resumos; 3) leitura integral do artigo. Foram adotados os seguintes critérios de elegibilidade para a inclusão dos artigos na presente revisão: 1) estudos de revisão e metanálise; 2) idioma inglês, português ou espanhol. Os estudos excluídos foram aqueles que não haviam compatibilidade com o assunto proposto, bem como os que não apresentavam informações conclusivas.

\section{RESULTADOS E DISCUSSÃO}

Analisar a comunidade microbiana permite identificar não só a composição, mas também sua estrutura e diversidade. A diversidade é subdividida em alfa e beta, esta é entre amostras e aquela é dentro da amostra (ARAOS; D'AGATA, 2019). A diversidade alfa é classificada de acordo com sua riqueza, uniformidade e relação filogenética, sendo importante para quantificar as mudanças na microbiota, seja por medicamentos, seja por doenças (ARAOS; D’AGATA, 2019). Já a diversidade beta estima a similaridade e a diferenciação entre grupos amostrais (ARAOS; D'AGATA, 2019). O microbioma humano consiste na microbiota total e nas biomoléculas associadas, em que ocorre a variabilidade interindividual (RUFF; GREILING; KRIEGEL, 2020). A idade, a dieta, a distribuição geográfica, o tipo de parto, as características da gestação, hospitalização e uso de medicações moldam a estrutura e composição do microbioma (ARAOS; D'AGATA, 2019). Como exemplo dessas peculiaridades individuais, pode citar que no intestino humano saudável os filos frequentemente encontrados são Bacterioidetes, Firmicutes, Actinobacteria e Proteobacteria, ademais, em indivíduos obesos predomina o filo Firmicutes, já nos magros, Bacterioidetes (ZORZO, 2017). Um exemplo de como os fatores ambientais podem influenciar a microbiota é o uso de antibióticos, que é um fator que induz mudanças drásticas na diversidade microbiana, principalmente no intestino anterior, devido ao aumento na abundância de patógenos potenciais e a redução de micróbios benéficos, além de influenciar na incidência e gravidade de doenças autoimunes e alérgicas (FITZGIBBON; MILLS, 2020; RUFF; GREILING; KRIEGEL, 2020). O uso de Claritromicina, Metronidazol e Omeprazol, por exemplo, causam alterações substanciais na composição do microbioma por até quatro anos (ARAOS; D'AGATA, 2019). Logo, o tratamento frequente com antimicrobianos têm um efeito negativo na homeostase imunológica e pode aumentar a suscetibilidade a doenças imunomediadas (FITZGIBBON; MILLS, 2020).

A composição das bactérias intestinais pode influenciar sobre o ganho de peso, inflamação e resistência à insulina no indivíduo, o que, consequentemente, aumenta o seu risco cardiometabólico (SUN; LI; NIE, 2020). Diversos mecanismo são estudados para explicar essa correlação, por exemplo, a relação do FastingInduced Adipose Factor (FIAF) e da lipase de lipoproteína (LPL); a questão envolvendo o monofosfato-adenosina proteína quinase ativada (AMP-Q); lipopolissacarídeos; e o eixo cérebro-intestino (ARAOS; D'AGATA, 2019). O intestino, o tecido adiposo e o fígado produzem a FIAF, que é responsável por inibir a LPL. A microbiota intestinal inibe a FIAF, o que, consequentemente, aumenta a atividade da LPL, que por sua vez determina maior absorção de ácidos graxos e acúmulo de triglicerídeos nas células do tecido adiposo (CRYAN; et al, 2019). A adenosina monofosfato (AMP), é responsável por ativar a via 5'monofosfato-adenosina proteína quinase (AMP-Q) (ABENAVOLI, et al., 2019). Essa via está inibida de forma que ativa os processos anabólicos e inibe os processos catabólicos, a fim de promover o ganho de massa (CRYAN; et al, 2019). Indivíduos magros, mesmo sobre dieta hipercalórica, conseguem promover a ativação da AMP$\mathrm{Q}$, entretanto, algumas pessoas, devido às características peculiares de suas microbiotas, não conseguem ativar a AMP-Q de forma a facilitar o ganho de massa. (ABENAVOLI, et al., 2019). Outrossim, o eixo intestino-cérebro também possui relação com o ganho de peso (MAN, 2018). Ácidos graxos se comportam como ligantes dos receptores FFAR2 (freefattyacids receptor 2) e FFAR3 (freefattyacids receptor 3) que se ligam à proteína $G$, os quais são expressos em células enteroendócrinas L e nas células do epitélio intestinal (BLISS; WHITESIDE, 2018). Essas células são responsáveis por produzir o peptídeo YY (PYY), que é um hormônio, cuja função é inibir a secreção e esvaziamento gástrico (MAN, 2018). Sendo assim, quando os receptores FFAR2 e FFAR3 estão ativos eles aumentam a produção de PYY, que por sua vez lentifica o trânsito no trato gastrointestinal de forma aumentar a absorção (BLISS; WHITESIDE, 2018).

O hormônio PYY também atua no SNC, inibindo os neurônios orexígenos localizados no núcleo arqueado e, consequentemente, induzem a saciedade (CRYAN; et al, 2019). Foi constatado em camundongos que aqueles que não apresentavam a via da proteína $\mathrm{G}$ denominada Gpr41, possuíam menor peso apesar do mesmo consumo de alimento (ABENAVOLI, et al., 2019). A explicação para esse fato seria que a diminuição da produção do peptídeo YY, aumenta a velocidade do trato gastrointestinal, diminuindo, consequentemente, a absorção (CRYAN; et al, 2019). Vale ressaltar que outra via intracelular ativada por esses receptores é a Gpr43, porém, essa não está envolvida no ganho de tecido adiposo, mas possivelmente ao processo inflamatório (ABENAVOLI, et al., 2019). A produção de ácidos graxos de cadeia curta intestinal é o principal fator relacionado ao aumento dos glucagon-like-peptide (GLP-1 e GLP-2) e PYY, bem como a diminuição da grelina, que é o hormônio produzido no estômago e intestino que atua no SNC promovendo estímulo dos neurônios orexígenos (MAN, 2018). Os lipopolissacarídeos (LPS) das bactérias da microbiota atuam como antígenos para os receptores Toll-Like receptores (TLR), quando estes são ativados nas células do sistema imune inato, liberam fatores pró-inflamatórios, como o fator de necrose tumoral alfa (TNF-alfa) e interleucinas (IL-1beta, IL-4 e IL-13) (BLISS; WHITESIDE, 2018). Esse estado inflamatório crônico e persistente está relacionado com o processo de resistência à insulina (MAN, 2018). Dessa forma, concentrações aumentadas de LPS, que depende do tipo de microbiota que o indivíduo possui, pode desencadear inflamação subclínica persistente, que participa da fisiopatologia de doenças, como diabetes mellitus, obesidade e outras (BLISS; WHITESIDE, 2018).

A microbiota intestinal regula a imunidade inata e adaptativa com consequências funcionais para a defesa do hospedeiro, seja contra patógeno, seja como tolerância imunológica a estímulos não patológicos (FUNG, 2020). A produção de interleucinas é reduzida na ausência da microbiota intestinal, consequentemente, influencia negativamente a imunidade do hospedeiro contra infecções (FITZGIBBON; MILLS, 2020). Os micróbios intestinais regulam o equilíbrio entre as células $\mathrm{T}$ auxiliares e as anti-inflamatórias, no intuito de garantir a homeostase imunológica, além disso, estão envolvidos na ativação de células $\mathrm{B}$ e na liberação subsequente de imunoglobulinas (FUNG, 2020). O desequilíbrio do microbioma é denominado disbiose, o qual pode ter influência ambiental, dietética, estilo de vida e medicamentosa (ARAOS; D'AGATA, 2019; FUNG, 2020). Uma microbiota disbiota pode se desenvolver durante a infância ou pode ser desencadeada por mudanças hormonais, por exemplo, além disso, ela pode ser transmitida às gerações subsequentes e contribuir para o rápido aumento das doenças imunomediadas (FUNG, 2020; RUFF; GREILING; KRIEGEL, 2020). As influências neonatais, mudanças hormonais e 
envelhecimento afetam as comunidades microbianas, o que contribui para o início, cronicidade e melhora de doenças imunomediadas (RUFF; GREILING; KRIEGEL, 2020). A disbiose pode acarretar em obesidade, doenças cardiovasculares, autoimunes, neurológicas, psiquiátricas, gastrointestinais, patologias malignas e infecções (ARAOS; D'AGATA, 2019; SUN; LI; NIE, 2020).

\section{CONCLUSÕES}

A individualidade do microbioma e a estabilidade funcional são essenciais para uma vida saudável. O microbioma, por meio de suas interações, molda a função imune e fisiológica local e extra-intestinal, como o sistema nervoso central do hospedeiro, e que a sua desregulação pode impulsionar patologias. Dessa forma, são necessários mais estudos para ampliar a compreensão de como se dão os processos fisiopatológicos envolvendo o microbioma na gênese das patologias. Outrossim, sabe-se que a microbiota intestinal de cada indivíduo é moldada por fatores genéticos e ambientais, logo, mudanças nos aspectos ambientais podem ser de grande valia no tratamento das patologias em que a microbioma está envolvida na sua etiologia.

\section{REFERÊNCIAS}

ABENAVOLI, L, et al. Gut Microbiota and Obesity: A Role for Probiotics. Nutrients, v. 11, 2019.

ARAOS, R.; D'AGATA, E.M.C. The human microbiota and infection prevention. Infection control and hospital epidemiology, v. 40, n. 5, p. 585-589, 2019.
BLISS, E.S; WHITESIDE, E. The Gut-Brain Axis, the Human Gut Microbiota and Their Integration in the Development of Obesity. Frontiers in Physiology, V. 9, 2018.

CRYAN, J.F., et al. The microbiota-gut-brain axis. Physiol Rev., v.99, p. 1877-2013, 2019.

FITZGIBBON, G.; MILLS, K.H.G. The microbiota and immunemediated diseases: opportunities for therapeutic intervention. European Journal of Immunology, v. 50, p. 326-337, 2020.

FUNG, T.C. The microbiota-immune áxis as a central mediator of gut-brain communication. Neurobiology of Disease: Elsevier, v. 136, p. 1-9, 2020.

MAN, S.M. Inflammasomes in the gastrointestinal tract: infection, cancer and gut microbiota homeostasis. NatureReviews, v. 15, 2018.

RAMIREZ, A.V.G. A importância da microbiota no organismo humano e sua relação com a obesidade. International Journal of Nutrology, V.10, N.4, P. 153-160, 2017.

RUFF, W.E.; GREILING, T.M.; KRIEGEL, M.A. Host-microbiota interactions in immune-mediated disease. Nature Reviews: Microbiology, v. 18, n. 9, p. 521-538, 2020.

SALOMÃO, J.O. et al. Implicações da microbiota intestinal humana no processo de obesidade e emagrecimento: revisão sistemática. Brazilian Journal of Health Review, Curitiba, 2020.

SOARES, D.K.N.S. Modulação da microbiota intestinal com probióticos e sua relação com a obesidade. REVISA, Valparaíso de Goiás, 2019.

SUN, L.J.; LI, J.N.; NIE, Y.Z. Gut hormones in microbiota-gut-brain cross-talk. Chinese Medical Journal, v. 133, n. 7, p. 826-833, 2020.

ZORZO, R.A. Impacto do microbioma intestinal no eixo cérebrointestino. Internacional Journal of Nutrology, v.10, n.1, p. 298$305,2017$. 